\title{
QUOTIENT RINGS OF NOETHERIAN MODULE FINITE RINGS
}

\author{
A. W. CHATTERS AND C. R. HAJARNAVIS
}

(Communicated by Lance W. Small)

\begin{abstract}
Let $R$ be a Noetherian ring which is a finite module over its centre $C$. We obtain a necessary and sufficient condition for $R$ to have a full quotient ring. We also show that when such a quotient ring does exist, it is obtained by inverting those elements of $C$ which are regular in $R$.
\end{abstract}

\section{INTRODUCTION}

The problem of deciding when a Noetherian ring possesses a classical ring of quotients was posed by Goldie [4]. Apart from the general characterisation given by Ore's theorem, this question is still open. The most significant result in this area so far has been that of Stafford [13], who obtained a criterion to determine when a Noetherian ring is its own quotient ring. Stafford also comments [13, p. 385] on the difficulty in tackling the more general problem of finding necessary and sufficient conditions for a Noetherian ring to have a full ring of quotients, pointing out that this question is intimately connected with that of localising at a prime ideal where an easy characterisation is unlikely. In this article we restrict our attention to those Noetherian rings which are finitely generated as modules over their centres. This class is a rich source of examples both with and without quotient rings $[2,11]$. Here the obstruction mentioned above does not apply since in this case an easy criterion given by Müller [10] to determine the localisibility of a prime ideal does exist.

Let $R$ be a Noetherian ring finitely generated as a module over its centre $C$. Let $\left\{P_{1}, \ldots, P_{n}\right\}$ be the set of regular primes in $R$ and $\mathscr{S}$ the set of all elements in $C$ which are regular in $R$. We show that $R$ has a full quotient ring if and only if $\left\{P_{1}, \ldots, P_{n}\right\}$ is precisely the set of primes lying over $\left\{P_{1} \cap C, \ldots, P_{n} \cap C\right\}$ (Proposition 3.3). This allows us to prove that the full quotient ring of $R$, when it exists, coincides with the partial quotient ring $R_{\mathscr{S}}$ (Theorem 3.4). In particular this gives us an easy test to apply to examples. It is normally straightforward to locate and invert $\mathscr{S}$ and obtain the ring $R_{\mathscr{S}}$. Theorem 3.4 shows that $R$ has a quotient ring if and only if $R_{\mathscr{S}}$ is its own quotient ring.

Received by the editors September 2, 1992.

1991 Mathematics Subject Classification. Primary 16U20. 
We also obtain a sufficiency criterion for $R$ to have a quotient ring in terms of the projective dimension of $R$ as a $C$-module. We prove that $R$ has a quotient ring if $R$ is torsionfree and has finite projective dimension as a $C$ module (Proposition 3.6).

The final section of the paper contains illustrative examples.

\section{NOTATION AND PRELIMINARIES}

Let $R$ be a not necessarily commutative ring and $I$ an ideal of $R$. We denote $\mathscr{C}(I)=\{c \in R \mid c+I$ is regular in the ring $R / I\}$. In particular, $\mathscr{C}(0)$ is the set of regular elements of $R$. When $X$ is a nonempty subset of a commutative ring $R, \operatorname{ann}(X)$ will denote the annihilator of $X$, i.e., $\operatorname{ann}(X)=\{r \in R \mid X r=0\}$.

The ring $Q$ is called a quotient ring if every regular element of $Q$ is a unit of $Q . Q$ is said to be the right quotient ring of $R$ if

(i) $R$ is a subring of $Q$;

(ii) every regular element of $R$ is a unit of $Q$; and

(iii) every element of $Q$ is expressible as $a c^{-1}$ for some $a, c \in R$ with $c$ regular.

For emphasis we sometimes refer to $Q$ as the full right quotient ring to distinguish it from partial quotient rings.

A multiplicatively closed set $\mathscr{X}$ of $R$ is said to be a right Ore set if given $a \in R$ and $c \in \mathscr{X}$ there exist $a_{1} \in R$ and $c_{1} \in \mathscr{X}$ such that $a c_{1}=c a_{1}$. It is well known that by Ore's theorem $R$ has a right quotient ring if and only if $\mathscr{C}(0)$ is a right Ore set.

The right quotient ring of $R$ coincides with the left quotient ring of $R$ when they both exist.

Throughout $R$ will denote a Noetherian ring module finite over its centre $C$, i.e., $R$ will be finitely generated as a $C$-module. In this situation $C$ is also a Noetherian ring by Eisenbud's theorem [8, Corollary 10.1.11]. In particular $R$ is an integral extension of $C$, and therefore by [1, Propositions $1.2,1.3$ and Corollary 1.7] the standard properties 'going up', 'lying over', and 'incomparability' hold between $R$ and $C$. Also for a prime ideal $\rho$ of $C$ there are only finitely many prime ideals of $R$ lying over $\mu$.

The term $R$ is $C$-torsion free will mean that for $0 \neq r \in R$ and $c$ regular in $C$ we have $r c \neq 0$.

Müller's theorem [10, Theorem 7 or 5, Theorems 11.20 and 12.21] provides us with the following convenient localisability criterion: Let $P$ be a prime ideal of $R$, and let $P=P_{1}, \ldots, P_{n}$ be the complete list of prime ideals lying over $P \cap C$. Then $\left\{P_{1}, \ldots, P_{n}\right\}$ is precisely the clique (clan) of $P$, and this clique is localisable; i.e., $\bigcap_{i=1}^{n} \mathscr{C}\left(P_{i}\right)$ is an Ore set.

By [12, Corollary 2.3 and Proposition 2.6] $R$ contains a unique, irredundant, finite set of primes $P_{1}, \ldots, P_{n}$ such that $\mathscr{C}(0)=\mathscr{C}\left(P_{1}\right) \cap \cdots \cap \mathscr{C}\left(P_{n}\right)$. These are called the regular primes of $R$.

$R$ is called a semilocal ring if $R / J$ is a semisimple Artinian ring where $J$ is the Jacobson radical of $R$. When $R$ is semilocal, $J$ is a finite intersection of the maximal ideals of $R$.

Where relevant the absence of the adjectives right or left will imply two-sided assumptions. 


\section{QUOTIENT RINGS}

Lemma 3.1. Let $R$ be a Noetherian ring which has a full quotient ring $Q$. Let $P_{1}, \ldots, P_{n}$ be the regular primes of $R$. Then $P_{i} \nsubseteq P_{j}$ for $i \neq j$.

Proof. By [13, Corollary 2.7] $Q$ is a semilocal ring. Let $M_{1}, \ldots, M_{k}$ be the maximal ideals of $Q$. Noting that units can be lifted over the Jacobson radical, we have $\mathscr{C}(0)=\mathscr{C}\left(M_{1}\right) \cap \cdots \cap \mathscr{C}\left(M_{k}\right)$ in $Q$. This implies that $\mathscr{C}(0)=$ $\mathscr{C}\left(M_{1} \cap R\right) \cap \cdots \cap \mathscr{C}\left(M_{k} \cap R\right)$ in $R$. It is easily seen that this intersection is not redundant. Clearly $M_{i} \cap R \nsubseteq M_{j} \cap R$ for $i \neq j$. By the uniqueness of regular primes it follows that $k=n$ and after suitable renumbering $P_{j}=M_{j} \cap R$ for $j=1, \ldots, n$.

Lemma 3.2. Let $R$ be a Noetherian ring which is module finite over its centre $C$. Suppose that $R$ has a full quotient ring. Let $P_{1}, \ldots, P_{n}$ be the regular primes of $R$. Then the set $\left\{P_{1}, \ldots, P_{n}\right\}$ contains all the prime ideals of $R$ which lie over the prime ideals $P_{1} \cap C, \ldots, P_{n} \cap C$ of $C$.

Proof. By assumption $\mathscr{C}(0)$ is an Ore set. An elementary argument shows that for each prime ideal $P$ of $R$ we have either $\mathscr{C}(0) \subseteq \mathscr{C}(P)$ or $\mathscr{C}(0) \cap P \neq \varnothing$. If $P$ and $Q$ are two primes in the same clique then we have $\mathscr{C}(0) \subseteq \mathscr{C}(P)$ if and only if $\mathscr{C}(0) \subseteq \mathscr{C}(Q)$ by [5, Lemma 12.17]. Take $Q$ to be a prime in the clique of $P_{1}$. We shall show that $Q=P_{i}$ for some $i$. This will imply that $\left\{P_{1}, \ldots, P_{n}\right\}$ is a union of cliques. We then have the result, noting that by Müller's theorem a clique in $R$ consists precisely of all primes lying over a fixed prime of $C$.

Suppose first that $Q \nsubseteq P_{i}$ for $1 \leq i \leq n$. Then $Q \cap \mathscr{C}\left(P_{i}\right) \neq \varnothing$. So, by [3, Lemma 13.4], $Q$ contains an element of $\bigcap_{i=1}^{n} \mathscr{C}\left(P_{i}\right)=\mathscr{C}(0)$. Thus $\mathscr{C}(0) \nsubseteq \mathscr{C}(Q)$, so by the argument above $\mathscr{C}(0) \nsubseteq \mathscr{C}\left(P_{1}\right)$. This is a contradiction since $\bigcap_{i=1}^{n} \mathscr{C}\left(P_{i}\right)=\mathscr{C}(0)$. Therefore, $Q \subseteq P_{i}$ for some $i$. Now since $Q$ belongs to the clique of $P_{1}$, by Müller's theorem we have $Q \cap C=P_{1} \cap C$. However, $Q \subseteq P_{i}$, so $P_{1} \cap C=Q \cap C \subseteq P_{i} \cap C$. Hence by 'going up' there exists a prime ideal $Q^{\prime}$ of $R$ such that $P_{1} \subseteq Q^{\prime}$ and $Q^{\prime} \cap C=P_{i} \cap C$. Now by Müller's theorem $Q^{\prime}$ belongs to the clique of $P_{i}$. So as with $Q$ and $P_{1}$ above, it follows that $Q^{\prime} \subseteq P_{j}$ for some $j$. Thus $P_{1} \subseteq Q^{\prime} \subseteq P_{j}$. We have $P_{1}=P_{j}$ by Lemma 3.1, so $P_{1}=Q^{\prime}$ and $P_{1} \cap C=P_{i} \cap C$. Therefore, $Q \cap C=P_{i} \cap C$ with $Q \subseteq P_{i}$. By 'incomparability' it follows that $Q=P_{i}$.

Proposition 3.3. Let $R$ be a Noetherian ring which is module finite over its centre $C$. Let $P_{1}, \ldots, P_{n}$ be the regular primes of $R$. Then $R$ has a full quotient ring if and only if the set $\left\{P_{1}, \ldots, P_{n}\right\}$ contains all the prime ideals of $R$ which lie over the prime ideals $P_{1} \cap C, \ldots, P_{n} \cap C$ of $C$.

Proof. If $R$ has a full quotient ring then Lemma 3.2 gives the result one way.

Conversely suppose that the set $\left\{P_{1}, \ldots, P_{n}\right\}$ satisfies the given condition. Then by Müller's theorem $\left\{P_{1}, \ldots, P_{n}\right\}$ is a union of cliques. Renumbering suitably, let $P_{1}, \ldots, P_{k}$ be a clique. Then these are all the primes lying over $P_{1} \cap C$. Let $a, c \in R$ with $c \in \mathscr{C}(0)$. We have $c \in \mathscr{C}\left(P_{i}\right)$ for all $i, 1 \leq i \leq n$, so $c \in \bigcap_{i=1}^{k} \mathscr{C}\left(P_{i}\right)$. Set $K=\{x \in R \mid a x \in c R\}$. Since the clique is localisable, $\bigcap_{i=1}^{k} \mathscr{C}\left(P_{i}\right)$ is an Ore set, so $K \cap \bigcap_{i=1}^{k} \mathscr{C}\left(P_{i}\right) \neq \varnothing$. This works for all the cliques in $\left\{P_{1}, \ldots, P_{n}\right\}$, so $K \cap \bigcap_{i=1}^{n} \mathscr{C}\left(P_{i}\right) \neq \varnothing$ by [3, Lemma 13.4]. Thus 
$K \cap \mathscr{C}(0) \neq \varnothing$, and $R$ has the right Ore condition with respect to $\mathscr{C}(0)$. The left Ore condition is analogous.

Theorem 3.4. Let $R$ be a Noetherian ring which is module finite over its centre $C$. Suppose that $R$ has a full quotient ring $Q$. Then $Q$ can be formed by inverting those elements of $C$ which are regular as elements of $R$.

Proof. Let $\mathscr{S}$ denote the elements of $C$ which are regular as elements of $R$. Let $R_{\mathscr{S}}$ be the partial quotient ring of $R$ formed by inverting the elements of $\mathscr{S}$. Then $R_{\mathscr{S}}$ is a subring of $Q$, and we shall prove the theorem by showing that $Q=R_{\mathscr{S}}$. It is clearly enough to show that $R_{\mathscr{S}}$ is a quotient ring. Note that $R_{\mathscr{S}}$ is a finitely generated module over its centre $C_{\mathscr{S}}$.

Let $P_{1}, \ldots, P_{n}$ be the regular primes of $R$. By Lemma 3.1 we know that $P_{i} \nsubseteq P_{j}$ for $i \neq j$. Also by Lemma 3.2 the set $\left\{P_{1}, \ldots, P_{n}\right\}$ contains all the prime ideals of $R$ which lie over any prime ideal of $C$ of the form $P_{i} \cap C$. So 'going up' gives $P_{i} \cap C \not \subset P_{j} \cap C$ for $i \neq j$. Clearly $\mathscr{S}=\{c \in C \mid c \notin$ $P_{i}$ for $\left.i=1, \ldots, n\right\}$. Hence $C_{\mathscr{S}}$ is a commutative Noetherian semilocal ring with maximal ideals the distinct members from the list $\left(P_{1} \cap C\right) C_{\mathscr{S}}, \ldots$, $\left(P_{n} \cap C\right) C_{\mathscr{S}}$. Now each $P_{i} R_{\mathscr{S}}$ is a prime ideal of $R_{\mathscr{S}}$ and lies over the maximal ideal $P_{i} R_{\mathscr{S}} \cap C_{\mathscr{S}}=\left(P_{i} \cap C\right) C_{\mathscr{S}}$ of $C_{\mathscr{S}}$. Since $R_{\mathscr{S}}$ is finite over $C_{\mathscr{S}}$, by 'incomparability', $P_{i} R_{\mathscr{S}}$ is a maximal ideal of $R_{\mathscr{S}}$.

Let $M$ be a maximal ideal of $R_{\mathscr{S}}$. We shall show that $M=P_{i} R_{\mathscr{S}}$ for some $i$. Now $M \cap R$ is a prime ideal of $R$, and by 'going up' $M \cap C_{\mathscr{S}}$ is a maximal ideal of $C_{\mathscr{S}}$. Hence $M \cap C_{\mathscr{S}}=\left(P_{i} \cap C\right) C_{\mathscr{S}}$ for some $i$. Therefore, $P_{i} \cap C \subseteq M \cap C$. We have $(M \cap R) R_{\mathscr{S}}=M \neq R_{\mathscr{S}}$; so $M \cap R \cap \mathscr{S}=\varnothing$. Thus $M \cap C \cap \mathscr{S}=\varnothing$. Hence $M \cap C \subseteq \bigcup_{j=1}^{n}\left(P_{j} \cap C\right)$. It follows by [6, Theorem 81] that $M \cap C \subseteq P_{j} \cap C$ for some $j$. Therefore, we have $P_{i} \cap C \subseteq M \cap C \subseteq P_{j} \cap C$. Since, as shown above, the inclusion cannot be strict, $P_{i} \cap C=M \cap C=P_{j} \cap C$. Thus $M \cap R$ lies over $P_{i} \cap C$. Hence, by Lemma 3.2, $M \cap R=P_{k}$ for some $k$. Therefore, $M=(M \cap R) R_{\mathscr{S}}=P_{k} R_{\mathscr{S}}$, and we have now shown that $P_{1} R_{\mathscr{S}}, \ldots, P_{n} R_{\mathscr{S}}$ are precisely the maximal ideals of $R_{\mathscr{S}}$.

Now let $a \in \mathscr{C}(0)$ in $R_{\mathscr{S}}$. Then as $\in R$ for some $s \in \mathscr{S}$. Clearly as is a regular element of $R$. Hence as $\in \mathscr{C}\left(P_{i}\right)$ in $R$ for all $i$ so that as $\in \mathscr{C}\left(P_{i} R_{\mathscr{S}}\right)$ in $R_{\mathscr{S}}$. Since $R_{\mathscr{S}}$ is a semilocal ring with maximal ideals $P_{1} R_{\mathscr{S}}, \ldots, P_{n} R_{\mathscr{S}}$, it follows that $a$ is a unit of $R_{\mathscr{S}}$. Therefore, $R_{\mathscr{S}}$ is a quotient ring, and $Q=R_{\mathscr{S}}$ as required.

Lemma 3.5. Let $R$ be a commutative Noetherian ring which is a quotient ring. Let $M$ be a finitely generated $R$-module of finite projective dimension. Then $M$ is projective.

Proof. The regular elements of $R$ are the elements which are regular modulo the maximal associated primes of the ideal $\{0\}$ in a primary decomposition of $\{0\}$. Thus $R$ is semilocal, and its maximal ideals are annihilator ideals. Let $P$ be a maximal ideal of $R$. It is easily seen that in the local ring $R_{P}$ the Jacobson radical is an annihilator. Hence, by [6, Theorem 191], $M_{P}=M \otimes_{R} R_{P}$ is a projective $R_{P}$-module. It follows by [9, Theorem 7.12] that $M$ is a projective $R$-module.

Proposition 3.6. Let $R$ be a Noetherian ring which is finitely generated, torsionfree, and of finite projective dimension over its centre $C$. Then $R$ has a full quotient ring which is a projective module over its centre. 
Proof. Let $\mathscr{S}$ be the set of all regular elements of $C$. Since $R$ is $C$-torsionfree, the elements of $\mathscr{S}$ are regular in $R$. We shall show that $R_{\mathscr{S}}$ is its own quotient ring and hence is the full quotient ring of $R$. Now $C_{\mathscr{S}}$ is the full quotient ring of $C$; it is also the centre of $R_{\mathscr{S}}$, and $R_{\mathscr{S}}$ has finite projective dimension as a $C_{\mathscr{S}}$-module. By Lemma 3.5 it follows that $R_{\mathscr{S}}$ is $C_{\mathscr{S}}$-projective and $C_{\mathscr{S}}$ is a semilocal ring. Let $1=e_{1}+\cdots+e_{n}$ be a sum of primitive orthogonal idempotents in $C_{\mathscr{S}}$. Then each $e_{i} R_{\mathscr{S}}$ is a finitely generated projective module over its centre $e_{i} C_{\mathscr{S}}$. Now $e_{i} C_{\mathscr{S}}$ is an indecomposable commutative Noetherian semilocal ring. So, by [7, Corollary 3.6 and Theorem 3.7], $e_{i} R_{\mathscr{S}}$ is a free $e_{i} C_{\mathscr{S}}$-module. Since $e_{i} C_{\mathscr{S}}$ is its own quotient ring, so also is $e_{i} R_{\mathscr{S}}$ by [13, Proposition 5.2]. Therefore, $R_{\mathscr{S}}$ is its own quotient ring as required.

Of course, $R$ has finite projective dimension over $C$ when $C$ is a Noetherian ring of finite global dimension. However, in this case $C$ is a semiprime ring, and then, if $R$ is a torsion free $C$-module, it is easy to see that $R$ has an Artinian quotient ring. A nontrivial situation when the above proposition is applicable is given in Example 4.2.

Note that as the examples in $[2,11]$ and Example 4.1 show, neither of the two conditions, 'finite projective dimension' and 'torsionfree' can be dropped to get the conclusion of Proposition 3.6.

\section{EXAMPLES}

Let $R$ be a Noetherian ring module finite over its centre $C$. Let $\mathscr{S}$ be the set of elements of $C$ which are regular as elements of $R$. We can always form the partial quotient ring $R_{\mathscr{S}}$ by inverting the elements of $\mathscr{S}$. Theorem 3.4 shows that $R_{\mathscr{S}}$ must be the quotient ring of $R$ if this exists. In particular when $C$ is its own quotient ring then $R_{\mathscr{S}}=R$ so that in this case either $R$ is a quotient ring or $R$ has no quotient ring at all. Our next example shows that the latter possibility can occur.

Example 4.1. Let $F$ be a field and $S=F[X, Y]$ the ring of polynomials in commuting indeterminates $X$ and $Y$. Let $T$ be the localisation of $S$ at the ideal generated by $X$ and $Y$. Consider the factor ring $C=T / Y(X T+Y T)$ of $T$. Set $y=Y+Y(X T+Y T), M=(X T+Y T) / Y(X T+Y T)$, and $P=y C$. Then $C$ is a commutative Noetherian ring with unique maximal ideal $M$, and $P$ is the only other prime ideal of $C$. We have $M=\operatorname{ann}(P)$. From this it follows that regular elements of $C$ are regular $\bmod (M)$ and hence are units of $C$. Thus $C$ is its own quotient ring. Let bars denote images in $\bar{C}=C / P$. Set

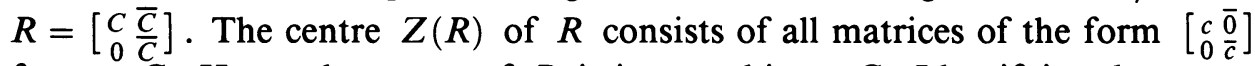
for $c \in C$. Hence the centre of $R$ is isomorphic to $C$. Identifying the centre with $C$, the ring $R$ is a finite module over $C$. Also $R$ is $C$-torsionfree since the regular elements of $C$ are units. By Theorem 3.4 we know that if $R$ had a quotient ring it would be $R$ itself. Now select $w$ to be a nonzero nonunit of $\bar{C}$. Then the matrix $\left[\begin{array}{ll}1 & \overline{0} \\ 0 & w\end{array}\right]$ is a regular element of $R$ but not a unit. Therefore, $R$ is not a quotient ring, so $R$ does not have a two-sided quotient ring. Note that $R$ does have a right quotient ring. Let $K$ be the quotient field of $\bar{C}$. Set $Q=\left[\begin{array}{ll}C & K \\ 0 & K\end{array}\right]$. Then $Q \neq R$, and $Q$ is the right quotient ring of $R$ but not the left quotient ring.

An element $\left[\begin{array}{ll}a & \bar{b} \\ 0 & \frac{c}{c}\end{array}\right]$ with $a, b, c \in C$ is regular in $R$ if and only if $a$ is a unit of $C$ and $\bar{C}$ is regular in $\bar{C}$. Thus the regular primes of $R$ are $P_{1}=\left[\begin{array}{c}M \\ 0\end{array}\right]$ 
and $P_{2}=\left[\begin{array}{ll}C & \bar{C} \\ 0 & \frac{0}{0}\end{array}\right]$. Set $\mu_{2}=Z(R) \cap P_{2}$. Then $\mu_{2}=\left[\begin{array}{l}P \\ 0 \\ 0\end{array}\right]$. Set $P_{3}=\left[\begin{array}{l}P \\ 0 \\ 0\end{array}\right]$. Then $P_{3}$ is a prime ideal of $R$, and $P_{3}$ lies over $\mu_{2}=Z(R) \cap P_{2}$. However, $P_{2}$ is a regular prime of $R$ whereas $P_{3}$ is not. Thus we can also see from Proposition 3.3 that $R$ does not possess a two-sided quotient ring.

Our next example gives a nontrivial application of Proposition 3.6.

Example 4.2. Let $A$ be any commutative Noetherian ring. Set $C=A[X, Y]$ the ring of polynomials in commuting indeterminates. Let $M=x C+y C$. It is easily seen that the projective dimension of $M$ cannot exceed 1 . We shall now show that $M$ is not projective as a $C$-module. Let $f: M \rightarrow C$ be a $C$ homomorphism. Since $f(x y)=f(x) y=f(y) x$ it follows that $f(x y) \in x y C$, and hence $f(x) \in x C$. Similarly $f(y) \in y C$, and we have $f(M) \subseteq M$. Now if $M$ were projective, the dual basis lemma would give $M=M^{2}$ which is a contradiction. Thus $M$ is a module of projective dimension 1 . Now set $R=\left[\begin{array}{ll}C & M \\ 0 & C\end{array}\right]$. Then $R$ is a Noetherian ring. Since $M$ is a faithful $C$-module, it is easily seen that the centre of $R$ consists of all matrices of the form $\left[\begin{array}{ll}c & 0 \\ 0 & c\end{array}\right]$ where $c \in C$ so that the centre of $R$ is isomorphic to $C$, and we shall identify it with $C$.

Thus $R$ is a Noetherian ring which is finitely generated, torsion-free, and of finite homological dimension over its centre $C$. Therefore, the hypotheses of Proposition 3.6 are satisfied with $C=A[X, Y]$ for any commutative Noetherian ring $A$.

In view of Müller's theorem, we can interpret Proposition 3.3 as follows: If $R$ is a Noetherian ring which is module-finite over its centre, then $R$ has a full quotient ring if and only if the set of regular primes of $R$ is closed under the formation of links. The following example shows that this is not true for general Noetherian rings.

Example 4.3. As in [5, Example 2ZC, p. 41], let $D$ be a Noetherian integral domain with a unique proper nonzero ideal $I$ such that $D / I$ is a field, say, $F$. Then $D$ does not satisfy the Ore condition with respect to $\mathscr{C}(I)$ since $I$ is an idempotent ideal and the elements of $\mathscr{C}(I)$ are regular in $D$. Set $R=\left[\begin{array}{ll}D & F \\ 0 & F\end{array}\right]$. Then $R$ is a Noetherian ring. The regular elements of $R$ have the form $\left[\begin{array}{ll}d & x \\ 0 & y\end{array}\right]$ where $d \in \mathscr{C}(I) \subseteq D$ and $x, y \in F$ with $y \neq 0$. Since $D$ does not satisfy the Ore condition with respect to $\mathscr{C}(I)$, it follows that $R$ does not have a full quotient ring. Also it is easily seen that the regular primes of $R$ are $P_{1}=\left[\begin{array}{ll}I & F \\ 0 & F\end{array}\right]$ and $P_{2}=\left[\begin{array}{ll}D & F \\ 0 & 0\end{array}\right]$. Note that $R / P_{1}$ and $R / P_{2}$ are both Artinian rings. (In fact, they are isomorphic to $F$ ). The only other prime ideal of $R$ is $P=\left[\begin{array}{l}0 \\ F \\ 0\end{array}\right]$. However, $R / P$ is not Artinian, so $P$ is not linked to either $P_{1}$ or $P_{2}$. Therefore the set $\left\{P_{1}, P_{2}\right\}$ of regular primes of $R$ is link-closed.

Added in proof. T. Lenagan has pointed out that in contrast to Example 4.3, the methods of this paper can easily be extended to prove the proposed extension of Proposition 3.3 to Noetherian rings satisfying the second layer condition.

\section{ACKNOWLEDGMENT}

It is a pleasure to thank Ken Goodearl and Lance Small for their helpful suggestions. 


\section{REFERENCES}

1. W. D. Blair, Right Noetherian rings integral over their centres, J. Algebra 27 (1973), 187-198.

2. A. W. Chatters, A non-singular Noetherian ring need not have a classical quotient ring, J. London Math. Soc. (2) 10 (1975), 66-68.

3. A. W. Chatters and C. R. Hajarnavis, Rings with chain conditions, Pitman Research Notes in Math., vol. 44, Longman Sci. Tech., Harlow, 1980.

4. A. W. Goldie, Some aspects of ring theory, Bull. London Math. Soc. 1 (1969), 129-154.

5. K. R. Goodearl and R. B. Warfield, Jr., An introduction to noncommutative Noetherian rings, London Math. Soc. Stud. Texts, vol. 16, Cambridge Univ. Press, Cambridge and New York, 1989.

6. I. Kaplansky, Commutative algebra, Allyn and Bacon, Boston, MA, 1970.

7. T. Y. Lam, Serre's conjecture, Lecture Notes in Math., vol. 635, Springer, Berlin, 1978.

8. J. C. McConnell and J. C. Robson, Noncommutative Noetherian rings, Wiley, Chichester, 1987.

9. H. Matsumura, Commutative ring theory, Cambridge Stud. Adv. Math., vol. 8, Cambridge Univ. Press, Cambridge and New York, 1986.

10. B. J. Müller, Localisation in noncommutative Noetherian rings, Canad. J. Math. 28 (1976), 600-610.

11. L. W. Small, Some questions in Noetherian rings, Bull. Amer. Math. Soc. 72 (1966), 853-857.

12. L. W. Small and J. T. Stafford, Regularity of zero divisors, Proc. London Math. Soc. (3) 44 (1982), 405-419.

13. J. T. Stafford, Noetherian full quotient rings, Proc. London Math. Soc. (3) 44 (1982), 385-404.

School of Mathematics, University Walk, Bristol BS8 1TW, England

Mathematics Institute, University of Warwick, Coventry CV4 7AL, ENGLAND

E-mail address: crh@maths .warwick. ac.uk 